\title{
Flavour Changing Neutral Currents, Weak-Scale Scalars and Rare Top Decays
}

\author{
Michael Luke and Martin J. Savage ${ }^{\dagger}$ \\ Department of Physics 0319 \\ University of California, San Diego \\ 9500 Gilman Drive \\ La Jolla, CA 92093-0319
}

\begin{abstract}
We examine the decays $t \rightarrow c \gamma$ and $c Z^{0}$ in the Standard Model with an extra scalar doublet and no discrete symmetry preventing tree-level flavour changing neutral currents. The Yukawa couplings of the new scalars are assumed to be proportional to fermion masses, evading bounds on FCNC's from the light quark sector. These rare top decays may be visible at the SSC.
\end{abstract}

UCSD/PTH 93-01

March 1993

$\dagger$ SSC Fellow 
Flavour changing neutral currents (FCNC's) involving the light quarks are known experimentally to be strongly suppressed, placing stringent bounds on the nature of new flavour physics. The gauge interaction of the fermions in the standard model has a global $[U(3)]^{5}$ flavour symmetry; this is broken to $[U(1)]^{4}$ by the Higgs Yukawa couplings, and the flavour symmetries are broken most strongly for the heavier quarks. If the observed pattern of masses and mixing angles reflects an underlying pattern of symmetry breaking, it is natural to expect that new flavour physics will also respect this pattern of symmetry breaking. Hence, it would couple most strongly to the top quark, for which the flavour symmetry is most strongly broken, while coupling only very weakly to the light fermions. This may allow the scale of new flavour physics to be as low as the weak scale without contradicting current experimental limits on FCNC's for light quarks.

A simple example is the Standard Model with extra scalars. These new scalars will typically have tree-level flavour-changing Yukawa couplings. However, as has been discussed by several authors [1]-[5], if the Yukawa couplings of the extra scalars are proportional to the fermion masses, low energy limits on FCNC's may be evaded because the flavour changing couplings to the light quarks are small. The discrete symmetries which are normally invoked in two-Higgs doublet models to forbid tree level FCNC's [6] are therefore unnecessary. It has been suggested that these FCNC's may be looked for in $b$ decays, and in particular the leptonic decay $B_{s} \rightarrow \mu^{+} \mu^{-}$may be observable at hadron colliders [2] [5].

If this philosophy is correct, one would expect to see the largest effects of these FCNC's in $t$ decays. The resulting rare $t$ decays may be searched for at the SSC, where $\sim 10^{8} \bar{t} t$ pairs are expected to be produced per year. FCNC's of gauge bosons in both the standard model [7]-12] and in two-Higgs doublet models with discrete symmetries [13]- 15] have been studied extensively in the past. In the Standard Model and two-Higgs doublet models (with ratios of Higgs vevs of order one) the branching ratios for $t \rightarrow c Z^{0}$ and $t \rightarrow c \gamma$ are less than $O\left(10^{-11}\right)$ [16] [17] and so would be unobservable at the SSC. In this paper we will calculate the rates for $t \rightarrow c \gamma$ and $t \rightarrow c Z^{0}$ in the Standard Model with an extra scalar doublet, but without the discrete symmetries which prevent tree-level FCNC's in two-Higgs doublet models. ${ }^{\dagger}$ We will not discuss the decay $t \rightarrow c g$ as the multi-jet background from the primary decay mode $t \rightarrow b W^{+}$will make this mode unobservable. We will assume that the new scalars are heavier than the top; the case of $m_{h}<m_{t}$ has been considered in [3], as has the tree-level decay $h \rightarrow \bar{t} c$ for $m_{h}>m_{t}$. We note that the latter process

$\dagger$ The mode $t \rightarrow c \gamma$ has also been recently discussed in [18]. 
( $h \rightarrow W+$ jets) has no clear signature and will be very difficult to see at the SSC; in this case loop effects such as those described here may be the only way to see these couplings at a hadron collider.

We consider a model with two scalar $S U(2)_{W}$ doublets, $\varphi_{1}$ and $\varphi_{2}$ :

$$
\varphi_{1}=\left(\begin{array}{c}
\varphi_{1}^{+} \\
\varphi_{1}^{0}
\end{array}\right), \quad\left(\begin{array}{c}
\varphi_{2}^{+} \\
\varphi_{2}^{0}
\end{array}\right)
$$

with Lagrangian

$$
\mathcal{L}_{\varphi}=\mathrm{D}^{\mu} \varphi_{1}^{\dagger} \mathrm{D}_{\mu} \varphi_{1}+\mathrm{D}^{\mu} \varphi_{2}^{\dagger} \mathrm{D}_{\mu} \varphi_{2}-V\left(\varphi_{1}, \varphi_{2}\right)
$$

where $V\left(\varphi_{1}, \varphi_{2}\right)$ is the most general potential consistent with the gauge symmetries. Since there is no global symmetry which distinguishes the two doublets we may work in a basis where only $\varphi_{1}$ has a VEV without loss of generality:

$$
\left\langle\varphi_{1}\right\rangle=\left(\begin{array}{c}
0 \\
v / \sqrt{2}
\end{array}\right), \quad\left\langle\varphi_{2}\right\rangle=0
$$

where $v=246 \mathrm{GeV}$. Three of the components of $\varphi_{1}$ become the longitudinal components of the $W^{ \pm}$and $Z^{0}$, while the spectrum contains the charged scalars $\varphi_{2}^{ \pm}$, the neutral scalars $h^{0}$ and $H^{0}$ and the pseudoscalar $A^{0}$, where

$$
\begin{aligned}
& H^{0}=\sqrt{2}\left[\left(\operatorname{Re} \varphi_{1}^{0}-v\right) \cos \alpha+\operatorname{Re} \varphi_{2}^{0} \sin \alpha\right], \\
& h^{0}=\sqrt{2}\left[-\left(\operatorname{Re} \varphi_{1}^{0}-v\right) \sin \alpha+\operatorname{Re} \varphi_{2}^{0} \cos \alpha\right], \\
& A^{0}=\sqrt{2}\left[-\operatorname{Im} \varphi_{2}^{0}\right] .
\end{aligned}
$$

The mixing angle $\alpha$ is determined by the potential and is a free parameter of the model, as are the masses $m_{\varphi_{2}^{ \pm}}, m_{h}, m_{H}$ and $m_{A}$. The Yukawa couplings to fermions are

$$
\begin{aligned}
\mathcal{L}_{Y}= & \lambda_{i j}^{U} \bar{Q}_{i} \tilde{\varphi}_{1} U_{j}+\lambda_{i j}^{D} \bar{Q}_{i} \varphi_{1} D_{j}+\lambda_{i j}^{E} \bar{L}_{i} \varphi_{1} E_{j} \\
& +\xi_{i j}^{U} \bar{Q}_{i} \tilde{\varphi}_{2} U_{j}+\xi_{i j}^{D} \bar{Q}_{i} \varphi_{2} D_{j}+\xi_{i j}^{E} \bar{L}_{i} \varphi_{2} E_{j}
\end{aligned}
$$

where $\tilde{\varphi}_{1,2} \equiv i \tau_{2} \varphi_{1,2}^{*}$. With the usual manipulations we write $\lambda^{U}, \lambda^{D}$ and $\lambda^{E}$ in terms of the mass matrices $\sqrt{2} M^{U} / v, \sqrt{2} M^{D} V^{\dagger} / v$ and $\sqrt{2} M^{E} / v$, where $V$ is the KobayashiMaskawa (KM) matrix. We have used all our freedom to redefine the fermion fields to diagonalise $M_{U}, M_{D} V^{\dagger}$ and $M_{E}$, so the matrices $\xi^{U, D, E}$ are general $3 \times 3$ matrices:

$$
\begin{aligned}
\xi^{U} & =\left(\begin{array}{lll}
\xi_{u u} & \xi_{u c} & \xi_{u t} \\
\xi_{c u} & \xi_{c c} & \xi_{c t} \\
\xi_{t u} & \xi_{t c} & \xi_{t t}
\end{array}\right) \quad \xi^{D}=\left(\begin{array}{lll}
\xi_{d d} & \xi_{d s} & \xi_{d b} \\
\xi_{s d} & \xi_{s s} & \xi_{s b} \\
\xi_{b d} & \xi_{b s} & \xi_{b b}
\end{array}\right) \\
\xi^{E} & =\left(\begin{array}{lll}
\xi_{e e} & \xi_{e \mu} & \xi_{e \tau} \\
\xi_{\mu e} & \xi_{\mu \mu} & \xi_{\mu \tau} \\
\xi_{\tau e} & \xi_{\tau \mu} & \xi_{\tau \tau}
\end{array}\right) .
\end{aligned}
$$


A priori, the $\xi$ 's are all free parameters, and are constrained in the light quark sector from the lack of observation of FCNC's. Two possible forms for the scalar couplings, motivated by the observed structures of the KM and fermion mass matrices, are the Cheng-Sher (CS) ansatz [1]

$$
\xi_{i j} \sim \frac{\sqrt{m_{i} m_{j}}}{v}
$$

and the Antaramian, Hall and Rasin (AHR) ansatz (for Higgs vevs $v_{1}=v_{2}=v$ ) [5]

$$
\begin{aligned}
\xi_{i j}^{U} & \sim \frac{m_{U_{j}}}{v}\left(\frac{m_{U_{i}} m_{D_{i}}}{m_{U_{j}} m_{D_{j}}}\right)^{1 / 4} \\
\xi_{i j}^{D} & \sim \frac{m_{D_{j}}}{v}\left(\frac{m_{U_{i}} m_{D_{i}}}{m_{U_{j}} m_{D_{j}}}\right)^{1 / 4} \\
\xi_{i j}^{E} & \sim \frac{\sqrt{m_{E_{i}} m_{E_{j}}}}{v} .
\end{aligned}
$$

In this work we will not make a specific ansatz for the $\xi$ 's but will simply assume that they are roughly proportional to powers of quark masses, allowing us to retain only $\xi_{t t}$ and the flavour changing couplings $\xi_{t c}$ and $\xi_{c t}$. In general the $\xi$ 's will also be complex, introducing additional $\mathrm{CP}$ violation into the theory; this will not be important for our results.

The general form of the amplitude $\mathcal{A}(t \rightarrow c+V)$ where $V=\gamma$ or $Z^{0}$ is

$$
\mathcal{A}=\frac{1}{16 \pi^{2}} \bar{u}_{c}\left(A \gamma^{\mu}+B \gamma^{\mu} \gamma_{5}+i C \sigma^{\mu \nu} \frac{q_{\nu}}{m_{t}}+i D \sigma^{\mu \nu} \gamma_{5} \frac{q_{\nu}}{m_{t}}\right) u_{t} \epsilon_{\mu},
$$

where $\bar{u}_{t}, u_{c}$ and $\epsilon_{\mu}$ are the incoming and outgoing spinors and the gauge boson polarisation vector respectively. The coefficient functions $A, B, C$ and $D$ are computed from the diagrams in fig. 1. There are contributions from both neutral and charged scalars in the intermediate states; note that the charged scalar couplings are not proportional to the KM matrix in this model. The expressions for the coefficient functions are lengthy and are given in the Appendix. For the photon, electromagnetic gauge invariance implies that the vector and axial vector form factors $A$ and $B$ vanish. In terms of the coefficient functions the decay widths are

$$
\begin{aligned}
\Gamma(t \rightarrow c \gamma) & =\frac{1}{8 \pi} m_{t}\left(|C|^{2}+|D|^{2}\right) \\
\Gamma\left(t \rightarrow c Z^{0}\right) & =\frac{1}{16 \pi m_{t}}\left(1-\frac{m_{Z}^{2}}{m_{t}^{2}}\right)\left(\frac{m_{t}^{2}}{m_{Z}^{2}}-1\right)\left[\left(m_{t}^{2}+2 m_{Z}^{2}\right)\left(|A|^{2}+|B|^{2}\right)\right. \\
& \left.-6 m_{Z}^{2} \operatorname{Re}\left(A^{*} C-B^{*} D\right)+m_{Z}^{2}\left(\frac{m_{Z}^{2}}{m_{t}^{2}}+2\right)\left(|C|^{2}+|D|^{2}\right)\right]
\end{aligned}
$$


from which we compute the branching fraction by normalising to the rate for $t \rightarrow b W$

$$
\Gamma(t \rightarrow b W)=\frac{G_{F}}{8 \pi \sqrt{2}}\left|V_{b t}\right|^{2} m_{t}^{3}\left(1-\frac{m_{W}^{2}}{m_{t}^{2}}\right)\left(1+\frac{m_{W}^{2}}{m_{t}^{2}}-2 \frac{m_{W}^{4}}{m_{t}^{4}}\right) .
$$

To avoid an excess of free parameters, we have plotted the branching ratios as a function of a common scalar mass, $m_{\varphi_{2}^{ \pm}}=m_{h}=m_{H}=m_{A} \equiv M$ in fig. 2. We have also set the flavour changing couplings of the charged scalars, neutral scalars and pseudoscalar to be equal to a generic coupling constant $\bar{\xi}_{t c}$, where $\bar{\xi}_{t c} \sim \xi_{t c} \sim \xi_{c t} \sim\left(\xi_{t c} \pm \xi_{c t}\right) / 2$. These simplifications do not qualitatively change our results. We have multiplied our branching fraction to $Z^{0}$ 's by the $6.7 \%$ branching fraction for $Z^{0} \rightarrow e^{+} e^{-}+\mu^{+} \mu^{-}$[19], as the hadronic decays of the $Z^{0}$ have an insurmountable background from the dominant two-body decay of the top $t \rightarrow b W \rightarrow b+$ hadrons.

For an ansatz of the form (7) we expect $\left(\xi_{t t} \bar{\xi}_{t c}\right)^{2} \sim m_{t}^{3} m_{c} / v^{4} \sim 10^{-3}$. The more complicated anzatz (8) gives a similar estimate. Given the expected $\sim 10^{8} \bar{t} t$ pairs expected per year at the SSC, this corresponds, for light scalars with masses of order the $t$ quark mass, to at best only a few events per year in the photon channel. For larger couplings, the expected signal of course dramatically improves.

It is useful to examine our results in the context of other limits on the scalar masses which have been discussed in the literature. The most stringent limits come from $\bar{B}^{0}-B^{0}$ and $\bar{K}^{0}-K^{0}$ mixing. For the AHR ansatz (8), these give lower limits $M \gtrsim 400 \mathrm{GeV}$ and $M \gtrsim 500 \mathrm{GeV}$ respectively [5]. In a recent work [4], Chang, Hou and Keung have calculated the two-loop contributions to $\mu \rightarrow e \gamma$ using the CS ansatz (7). From the current upper limits on this decay mode they placed a lower bound of $M \gtrsim 200 \mathrm{GeV}$. Other processes place lower limits on $M$ which are less than the $t$ quark mass and hence do not constrain our results. It is important to note, as pointed out in [2] and [4], that these constraints arise from couplings to the down-type quark and lepton sectors only. It is conceivable that flavour changing couplings are large only in the up-type quark sector, for which the constraints on $M$ are much weaker. The possibility of $M \sim m_{t}$ with large flavour changing couplings in the up sector is not excluded. In the absence of any compelling theoretical reason to prefer a particular choice of $\xi^{U}$ 's we feel it is best to simply regard them as free parameters to be measured or constrained at the SSC.

In conclusion, we have calculated the branching ratios for $t \rightarrow c \gamma$ and $t \rightarrow c Z^{0}$ in the Standard Model with an extra scalar doublet, but without discrete symmetries preventing tree-level FCNC's. For flavour changing couplings suggested by simple ansatzes 
for the Yukawa couplings these decay modes would not be observable at the SSC, given the constraints on the scalar masses from other processes. However, these constraints may be evaded if flavour changing couplings are larger in the $u$ quark sector than for the $d$ quark and lepton sectors. In this case, the flavour changing couplings and scalar masses are virtually unconstrained. Consequently, sizeable branching ratios for $t \rightarrow c \gamma$ and $t \rightarrow c Z^{0}$ are possible. To determine whether or not these decays are observable at the SSC requires a thorough background study; we have not attempted this in this work.

We wish to thank Ann Nelson and David Kaplan for numerous discussions. This work was supported in part by DOE grant DE-FG03-90ER40546 and by National Science Foundation Grant PHY-8958081. MJS acknowledges the support of a Superconducting Supercollider National Fellowship from the Texas National Research Laboratory Commission under grant FCFY9219.

\section{Appendix A. Expressions for the Coefficient Functions}

For simplicity, we set the mixing angle $\alpha$ to zero; the flavour changing couplings then only involve $\varphi_{2}$. We split each coefficient up into contributions from graphs with neutral scalars, graphs with pseudoscalars, graphs with both neutral scalars and pseudoscalars and finally graphs with charged scalars: $A=A^{h}+A^{A}+A^{M}+A^{C}$. The contribution from graphs with only neutral scalars is given by

$$
\begin{aligned}
A^{h}= & \int_{0}^{1} d x\left(-x \log \frac{\beta^{h}}{\mu^{2}}+\log \gamma^{h}\right)\left(\xi_{V}^{h} a_{V}-\xi_{A}^{h} a_{A}\right) \\
& +\int d x d y\left[\left(1+\log \frac{\eta^{h}}{\mu^{2}}-\frac{x y k^{2}}{\eta^{h}}\right)\left(\xi_{V}^{h} a_{V}+\xi_{A}^{h} a_{A}\right)\right. \\
& \left.+\frac{m_{t}^{2}(x+y-2)}{\eta^{h}}\left(\xi_{V}^{h} a_{V}-\xi_{A}^{h} a_{A}\right)\right] \\
B^{h}= & \int_{0}^{1} d x\left(-x \log \frac{\beta^{h}}{\mu^{2}}+\log \gamma^{h}\right)\left(\xi_{V}^{h} a_{A}-\xi_{A}^{h} a_{V}\right) \\
& +\int d x d y\left[\left(-1-\log \frac{\eta^{h}}{\mu^{2}}+\frac{x y k^{2}}{\eta^{h}}\right)\left(\xi_{V}^{h} a_{A}+\xi_{A}^{h} a_{V}\right)\right. \\
& \left.+\frac{m_{t}^{2}(x+y-2)}{\eta^{h}}\left(\xi_{V}^{h} a_{A}-\xi_{A}^{h} a_{V}\right)\right] \\
C^{h}= & m_{t}^{2} \int d x d y \frac{x}{\eta^{h}}\left(\xi_{V}^{h} a_{V}-\xi_{A}^{h} a_{A}\right)+\frac{2 y-x y-y^{2}}{\eta^{h}}\left(\xi_{V}^{h} a_{V}+\xi_{A}^{h} a_{A}\right) \\
D^{h}= & m_{t}^{2} \int d x d y \frac{x}{\eta^{h}}\left(-\xi_{V}^{h} a_{A}+\xi_{A}^{h} a_{V}\right)+\frac{2 y-x y-y^{2}}{\eta^{h}}\left(\xi_{V}^{h} a_{A}+\xi_{A}^{h} a_{V}\right)
\end{aligned}
$$


where we have defined the functions

$$
\begin{aligned}
\beta^{h} & =(1-x)^{2} m_{t}^{2}+x m_{h}^{2} \\
\gamma^{h} & =\frac{(1-x) m_{t}^{2}+x m_{h}^{2}}{(1-x)^{2} m_{t}^{2}+x m_{h}^{2}} \\
\eta^{h} & =(1-x-y) m_{h}^{2}+\left(x+x y+y^{2}\right) m_{t}^{2}-x y k^{2}+i \epsilon
\end{aligned}
$$

and the couplings

$$
a_{V}^{\gamma}=\frac{2 e}{3}, \quad a_{A}^{\gamma}=0, \quad a_{M}^{\gamma}=0
$$

for the photon,

$$
a_{V}^{Z}=\frac{g}{4 \cos \theta_{W}}\left(1-\frac{8}{3} \sin \theta_{W}^{2}\right), \quad a_{A}^{Z}=-\frac{g}{4 \cos \theta_{W}}, \quad a_{M}^{Z}=\frac{g}{2 \cos \theta_{W}}
$$

for the $Z^{0}$. The products of Yukawa couplings are

$$
\xi_{V}^{h}=\xi_{t t}\left(\xi_{c t}+\xi_{t c}^{*}\right) / 4, \quad \xi_{A}^{h}=\xi_{t t}\left(\xi_{c t}-\xi_{t c}^{*}\right) / 4
$$

Finally, $k^{2}$ is the mass of the gauge boson and $\mu$ is the renormalisation scale. The sum of the graphs is of course finite and the result independent of $\mu$.

The contribution from graphs with only neutral pseudoscalars is given by

$$
\begin{aligned}
A^{A}= & \int_{0}^{1} d x\left(-x \log \frac{\beta^{A}}{\mu^{2}}-\log \gamma^{A}\right)\left(\xi_{V}^{A} a_{V}-\xi_{A}^{A} a_{A}\right) \\
& +\int d x d y\left[\left(1+\log \frac{\eta^{A}}{\mu^{2}}+\frac{x y k^{2}}{\eta^{A}}\right)\left(\xi_{V}^{A} a_{V}-\xi_{A}^{A} a_{A}\right)\right. \\
& \left.\quad-\frac{m_{t}^{2}(x+y)}{\eta^{A}}\left(\xi_{V}^{A} a_{V}+\xi_{A}^{A} a_{A}\right)\right] \\
B^{A}= & \int_{0}^{1} d x\left(-x \log \frac{\beta^{A}}{\mu^{2}}-\log \gamma^{A}\right)\left(\xi_{V}^{A} a_{A}+\xi_{A}^{A} a_{V}\right) \\
& +\int d x d y\left[\left(-1-\log \frac{\eta^{A}}{\mu^{2}}-\frac{x y k^{2}}{\eta^{A}}\right)\left(\xi_{V}^{A} a_{A}-\xi_{A}^{A} a_{V}\right)\right. \\
& \left.\quad-\frac{m_{t}^{2}(x+y)}{\eta^{A}}\left(\xi_{V}^{A} a_{A}+\xi_{A}^{A} a_{V}\right)\right] \\
C^{A}= & m_{t}^{2} \int d x d y \frac{-x}{\eta^{A}}\left(\xi_{V}^{A} a_{V}+\xi_{A}^{A} a_{A}\right)-\frac{y(x+y)}{\eta^{A}}\left(\xi_{V}^{A} a_{V}-\xi_{A}^{A} a_{A}\right) \\
D^{A}= & m_{t}^{2} \int d x d y \frac{x}{\eta^{A}}\left(\xi_{V}^{A} a_{A}+\xi_{A}^{A} a_{V}\right)-\frac{y(x+y)}{\eta^{A}}\left(\xi_{V}^{A} a_{A}-\xi_{A}^{A} a_{V}\right)
\end{aligned}
$$


where

$$
\begin{aligned}
\beta^{A} & =(1-x)^{2} m_{t}^{2}+x m_{A}^{2} \\
\gamma^{A} & =\frac{(1-x) m_{t}^{2}+x m_{A}^{2}}{(1-x)^{2} m_{t}^{2}+x m_{A}^{2}} \\
\eta^{A} & =(1-x-y) m_{A}^{2}+\left(x+x y+y^{2}\right) m_{t}^{2}-x y k^{2}+i \epsilon
\end{aligned}
$$

and

$$
\xi_{V}^{A}=\xi_{t t}\left(\xi_{c t}-\xi_{t c}^{*}\right) / 4, \quad \xi_{A}^{A}=\xi_{t t}\left(\xi_{c t}+\xi_{t c}^{*}\right) / 4 .
$$

The graphs involving both the scalar and pseudoscalar particles give:

$$
\begin{array}{r}
A^{M}=a_{M} \int d x d y\left(\xi_{A}^{M 1}\left(-\log \frac{\eta^{M 1}}{\mu^{2}}+\frac{m_{t}^{2}\left(1-y-x y-x^{2}\right)}{\eta^{M 1}}\right)\right. \\
\left.+\xi_{A}^{M 2}\left(\log \frac{\eta^{M 2}}{\mu^{2}}+\frac{m_{t}^{2}\left(1-y+x y-2 x+x^{2}\right)}{\eta^{M 2}}\right)\right) \\
B^{M}=a_{M} \int d x d y\left(\xi_{V}^{M 1}\left(-\log \frac{\eta^{M 1}}{\mu^{2}}+\frac{m_{t}^{2}\left(1-y-x y-x^{2}\right)}{\eta^{M 1}}\right)\right. \\
\left.\quad-\xi_{V}^{M 2}\left(\log \frac{\eta^{M 2}}{\mu^{2}}+\frac{m_{t}^{2}\left(1-y+x y-2 x+x^{2}\right)}{\eta^{M 2}}\right)\right) \\
C^{M}=a_{M} m_{t}^{2} \int d x d y \xi_{A}^{M 1} \frac{1-y-x y-x^{2}}{\eta^{M 1}}+\xi_{A}^{M 2} \frac{1-y+x y-2 x+x^{2}}{\eta^{M 2}} \\
D^{M}=-a_{M} m_{t}^{2} \int d x d y \xi_{V}^{M 1} \frac{1-y-x y-x^{2}}{\eta^{M 1}}-\xi_{V}^{M 2} \frac{1-y+x y-2 x+x^{2}}{\eta^{M 2}}
\end{array}
$$

where

$$
\begin{aligned}
& \eta^{M 1}=\left(1-2 x-y+x^{2}+x y\right) m_{t}^{2}+x m_{h}^{2}+y m_{A}^{2}-x y k^{2}+i \epsilon \\
& \eta^{M 2}=\left(1-2 x-y+x^{2}+x y\right) m_{t}^{2}+x m_{A}^{2}+y m_{h}^{2}-x y k^{2}+i \epsilon
\end{aligned}
$$

and

$$
\begin{aligned}
& \xi_{V}^{M 1}=\xi_{t t}\left(\xi_{c t}-\xi_{t c}^{*}\right) / 4, \quad \xi_{A}^{M 1}=\xi_{t t}\left(\xi_{c t}+\xi_{t c}^{*}\right) / 4 \\
& \xi_{V}^{M 2}=\xi_{t t}\left(\xi_{c t}+\xi_{t c}^{*}\right) / 4, \quad \xi_{A}^{M 2}=\xi_{t t}\left(\xi_{c t}-\xi_{t c}^{*}\right) / 4 .
\end{aligned}
$$

Finally, the contribution from graphs involving the charged scalar is

$$
\begin{aligned}
& A^{C}=B^{C}=\xi^{C}\left[-4 a_{R} \int_{0}^{1} d x x \log \beta^{C}\right. \\
&+4 b_{L}\left(\frac{1}{2}+\int d x d y\left[\log \frac{\eta^{C 1}}{\mu^{2}}-\frac{x y k^{2}}{\eta^{C 1}}\right]\right) \\
&\left.+2 j_{c} \int d x d y\left(\log \frac{\eta^{C 2}}{\mu^{2}}-\frac{m_{t}^{2} y(1-x-y)}{\eta^{C 2}}\right)\right] \\
& C^{C}=-D^{C}=\xi^{C} m_{t}^{2} \int d x d y y(1-x-y)\left(\frac{4 b_{L}}{\eta^{C 1}}-\frac{2 j_{c}}{\eta^{C 2}}\right)
\end{aligned}
$$


where ,

$$
\begin{aligned}
& \xi^{C}=\xi_{t t} \xi_{t c}^{*} / 4 \\
& \beta^{C}=x\left(m_{\varphi_{2}^{ \pm}}^{2}-(1-x) m_{t}^{2}\right) \\
& \eta^{C 1}=(1-x-y)\left(m_{\varphi_{2}^{ \pm}}^{2}-x m_{t}^{2}\right)-x y k^{2}+i \epsilon \\
& \eta^{C 2}=(x+y) m_{\varphi_{2}^{ \pm}}^{2}-y(1-x-y) m_{t}^{2}-x y k^{2}+i \epsilon
\end{aligned}
$$

and the couplings are

$$
a_{R}^{\gamma}=\frac{e}{3}, \quad b_{L}^{\gamma}=-\frac{e}{6}, \quad j_{c}^{\gamma}=e
$$

for the photon and

$$
a_{R}^{Z}=-\frac{g s_{W}^{2}}{3 c_{W}}, \quad b_{L}^{Z}=-\frac{g}{4 c_{W}}\left(1-\frac{2}{3} s_{W}^{2}\right), \quad j_{c}^{Z}=\frac{g}{2 c_{W}}\left(1-2 s_{W}^{2}\right)
$$

for the $Z^{0}$. 


\section{References}

[1] T. P. Cheng and M. Sher, Phys. Rev. D44 (1991) 1461;

Phys. Rev. D35 (1987) 3484.

[2] M.J. Savage, Phys. Lett. 266B (1991) 135.

[3] W.-S. Hou, Phys. Lett. 296B (1992) 179.

[4] D. Chang, W.S. Hou and W.Y Keung, CERN-TH.6795/93 (1993).

[5] A. Antaramian, L.J. Hall and A. Rasin, Phys. Rev. Lett. 69 (1992) 1871.

[6] S. Glashow and S. Weinberg, Phys. Rev. D15 (1977) 1958.

[7] V. Ganapathi, T. Weiler, E. Laermann, I. Schmitt and P.M. Zerwas, Phys. Rev. 27 (1983) 579

[8] G. Mann and T. Riemann, Annalen der Physik, 7 (1983) 334

[9] M. Clements, C. Footman, A. Kronfeld, S. Narasimhan and D. Photiadis, Phys. Rev. D27 (1983) 570

[10] A. Axelrod, Nuc. Phys. B209 (1982) 349

[11] E. Ma and A. Pramudita, Phys. Rev. D22 (1980) 214

[12] M.J. Duncan, Phys. Rev. D31 (1985) 1139

[13] M.J. Savage, CALT-68-1496, (1988)

[14] C. Busch, Nuc. Phys. B319 (1989) 15

[15] J. Hewett, S. Nandi and T. Rizzo, Phys. Rev. D39 (1989) 250

[16] B. Grzadkowski, J. F. Gunion and P. Krawczyk, Phys. Lett. 268B (1991) 106

[17] G. Eilam, J.L. Hewett and A. Soni, Phys. Rev. D44 (1991) 1473

[18] J. L. Diaz-Cruz and G. Lopez Castro, "CP Violation and FCNC with the Top Quark", to appear in Phys. Lett. B.

[19] Particle Data Group, Phys. Rev. D45 (1992) S1. 


\section{Figure Captions}

Fig. 1. Feynman diagrams contributing to the decays $t \rightarrow c \gamma$ and $t \rightarrow c Z^{0}$

Fig. 2. Branching fraction for $t \rightarrow c \gamma$ and $t \rightarrow c Z^{0} \rightarrow c e^{+} e^{-}+c \mu^{+} \mu^{-}$divided by the product of coupling constants $\left(\xi_{t t} \bar{\xi}_{t c}\right)^{2}$ plotted as a function of a common scalar mass. The solid, dashed and dot-dashed curves correspond to a top quark mass of $110,150,180 \mathrm{GeV}$ respectively. 
Figure 1
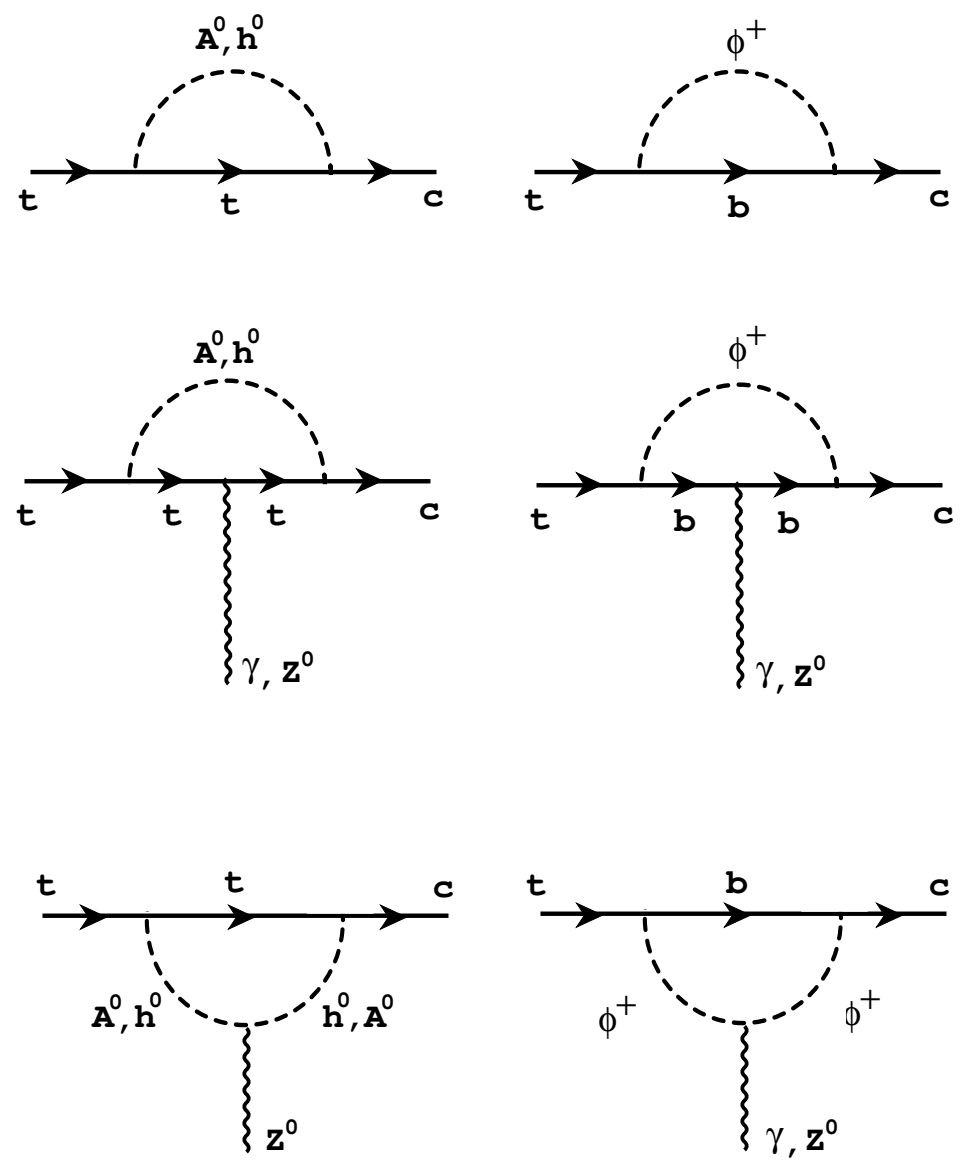
Figure 2
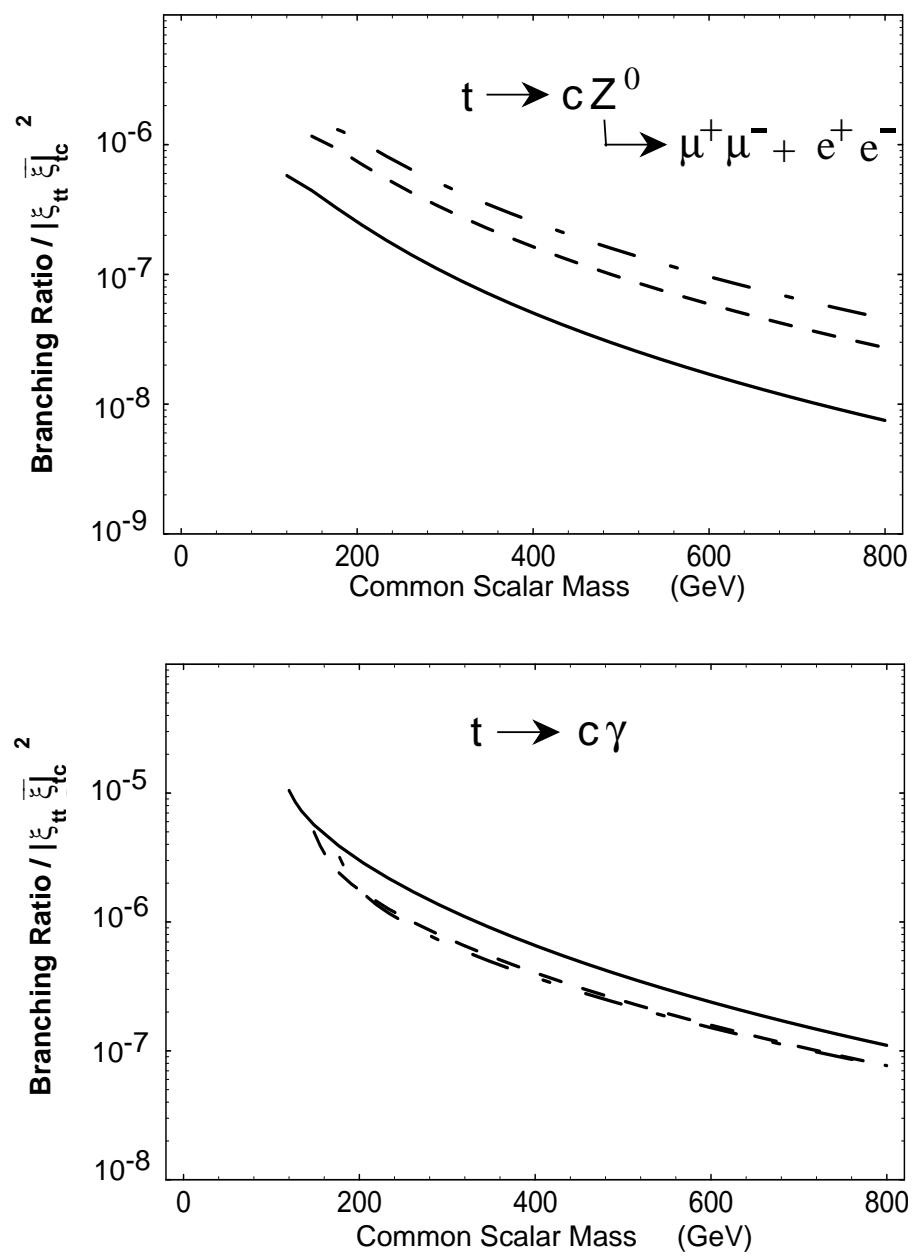